\title{
Outflow Propagation in Collapsars: Collimated Jets and Expanding Outflows
}

\author{
Akira Mizuta - Tatsuya Yamasaki . \\ Shigehiro Nagataki $\cdot$ Shin Mineshige
}

\begin{abstract}
We investigate the outflow propagation in the collapsar in the context of gamma-ray bursts (GRBs) with 2D relativistic hydrodynamic simulations. We vary the specific internal energy and bulk Lorentz factor of the injected outflow from non-relativistic regime to relativistic one, fixing the power of the outflow to be $10^{51} \mathrm{erg} \mathrm{s}^{-1}$. We observed the collimated outflow, when the Lorentz factor of the injected outflow is roughly greater than 2. To the contrary, when the velocity of the injected outflow is slower, the expanding outflow is observed. The transition from collimated jet to expanding outflow continuously occurs by decreasing the injected velocity. Different features of the dynamics of the outflows would cause the difference between the GRBs and similar phenomena, such as, X-ray flashes.
\end{abstract}

Keywords Hydrodynamics · Jet · GRBs · Supernovae · Shock $\cdot$ Relativity

\section{Introduction}

The gamma-ray bursts (GRBs) are the most energetic phenomena in the sky. A collimated and relativistic jet is

\footnotetext{
A. Mizuta $(\square)$

Max-Planck-Institute für Astrophysik, Karl-Schwarzschild-Str. 1, 85741 Garching, Germany

e-mail: mizuta@MPA-Garching.MPG.DE

T. Yamasaki $\cdot$ S. Mineshige

Yukawa Institute for Theoretical Physics, Kyoto University, Oiwake-cho, Kitashirakawa, Sakyo-ku, Kyoto 606-8502, Japan

S. Nagataki

Yukawa Institute for Theoretical Physics, Kyoto University, Oiwake-cho, Kitashirakawa, Sakyo-ku, Kyoto 606-8502, Japan; KIPAC, Stanford University, P.O.Box 20450, MS 29, Stanford, CA, 94309, USA
}

necessary to explain the observational features of GRBs (Piran, 2000). The central engine of the GRBs is not fully understood yet. However, recent observations of the long duration GBRs associated with $\mathrm{SNe}$, for example, GRB980425/SN1998bw (Galama et al., 1998; Iwamoto et al., 1998) and GRB030329/SN2003dh (Hjorth et al., 2003; Price et al., 2003; Stanek et al., 2003) link the GRBs and the death of massive stars. Note, both SN1998bw and SN2003dh are categorized to a sub-class of the $\mathrm{SNe}$, such as, hypernovae whose explosion energy is $\sim 10^{52}$ ergs which is one order magnitude higher than that of normal supernova explosion.

The similar phenomena called as X-ray flashes (XRFs) are also observed (Heise et al., 2001). XRFs have larger fluence in the X-ray band than in the gamma-ray. Since the event rate of XRFs is similar to GRBs, several hypotheses are proposed to link these events. Nakamura (2000) proposed an unified model that explains the different properties of GRBs and XRFs by the different viewing angle of the collimated outflow. Lamb et al. (2005) proposed a model that explains the different properties by the different opening angle of the outflow.

Theoretically the relation between the death of the massive stars and GRBs was predicted by Woosley (1993). That is so called collapsar model. When an iron core of a rapidly rotating massive star collapses, a proto neutron star or black hole is formed in the center of the progenitor. Though the gas along the rotational axis can freefall quickly, the gas along the equatorial plane gradually falls into the center because of the large centrifugal force. As a result an accretion disk is formed. MacFadyen and Woosley (1999) performed hydrodynamic simulations of this model. They deposit thermal energy in the polar region around the core, assuming neutrino emission from the accretion disk, and neutrino and anti-neutrino annihilation there. Then the gas expands and forms an bipolar flow. Since the calculation was Newtonian 
one, the relativistic effects which are important for GRBs are not included. Aloy et al. (2000) did relativistic hydrodynamic simulations of the same type of problems done by MacFadyen and Woosley (1999). They showed collimated and relativistic jet along the polar axis of the progenitor. The jet finally breaks out from the progenitor, making a highly Lorentz factor component up to $\Gamma \sim 40$.

Another type of relativistic hydrodynamic simulations have also done by Zhang et al. (2003, 2004), and Umeda et al. (2005). They inject not only a thermal energy but also a kinetic energy from the computational boundary, assuming an outflow formation around the center of the progenitor. They followed the outflow propagation in the progenitor and interstellar medium. All their model were initially outflows with a large thermal energy and showed successful eruption from the progenitor, i.e., relativistic jets. But there still remain some issues on the propagation of the outflows in the progenitor. Which type of the outflow can keep the collimated structure and how do they keep the good collimation. In this paper, we show the different types of outflows in the collapsar.

\section{Model}

We study the outflow propagation in the progenitor, assuming an outflow formation after the core collapse. The radial mass profile of the progenitor developed by Hashimoto (1995) is used. The progenitor had a mass of about 40 solar masses in the main sequence and has 16 solar masses in the pre-supernovae stage. The radius of the progenitor is $3.7 \times$ $10^{10} \mathrm{~cm}$. We use non-uniform grid points, assuming the axisymmetric geometry $(r-z)$. Logarithmically uniform 500 grid points are spaced for $2 \times 10^{8} \mathrm{~cm}<z<6.6 \times 10^{10} \mathrm{~cm}$. We also set uniform 120 zones for $0<r<1.2 \times 10^{9} \mathrm{~cm}$ and logarithmically uniform 130 zones for $1.2 \times 10^{9}<r<$ $1.1 \times 10^{10} \mathrm{~cm}$. The inner boundary of the computational box is located at the distance of $2 \times 10^{8} \mathrm{~cm}$ from the center of the progenitor. In this study the origin of the coordinate corresponds to the center of the progenitor. The boundary conditions at the cylindrical axis $(r=0)$ and $z=2 \times 10^{8} \mathrm{~cm}$, are reflective one except $0<r<7 \times 10^{7} \mathrm{~cm}$ at $z=2 \times 10^{8} \mathrm{~cm}$ where an outflow is injected. The boundary conditions at other boundaries are outflow boundary condition. The mass densities of the progenitor is $\sim 10^{6} \mathrm{~g} \mathrm{~cm}^{-3}$ (around the inner boundary), $\sim 1 \mathrm{~g} \mathrm{~cm}^{-3}$ (at the surface of the progenitor), and $10^{-6} \mathrm{~g} \mathrm{~cm}^{-3}$ (constant outside of the progenitor).

The 2D special relativistic hydrodynamic equations are solved, using our relativistic hydrodynamic code based on Godunov-type scheme (Mizuta et al., 2004, 2006). An ideal equation of state $p=(\gamma-1) \rho \epsilon$ is also solved to close the equations, where $p$ is pressure, the constant $\gamma(=4 / 3)$ is specific heat ratio, $\rho$ is rest mass density, and $\epsilon$ is specific internal energy. As our current numerical code can handle only constant specific heat ratio, we take precedence the state for the relativistic temperature $\gamma(=4 / 3)$ in this paper. Since the timescale for the outflows to cross the progenitor is much shorter than that of the freefall of the envelopes, we ignore the gravitational potential by the formed black hole or proto neutron star at the center of the progenitor. The initial gas temperature of the envelope and outside of the surface is set to be very low $\left(\epsilon / c^{2}=10^{-9}\right.$ and $\left.\epsilon / c^{2}=10^{-6}\right)$.

We assume an outflow formation from the center of the progenitor. It is also assumed that the outflow is parallel to the cylindrical axis. We inject this outflow from the boundary described above. Four parameters are necessary to define the outflow condition. In this paper, we fixed two of them. The first one is the power of the outflow which is fixed to be $10^{51} \mathrm{ergs} \mathrm{s}^{-1}$. The total energy by ten seconds injection satisfies $10^{52}$ erg which is the energy of the hypernova explosion. The second one is the radius of the injected outflow which is fixed to be $7 \times 10^{7} \mathrm{~cm}$. We vary other two parameters, such as, the specific internal energy $\epsilon_{0}$ and the bulk Lorentz factor $\Gamma_{0}$, where subscripts ' 0 ' stand for the values of the injected outflows from the computational boundary. The bulk Lorentz factor is varied from $\Gamma_{0}=1.05$ to $\Gamma_{0}=5$, corresponding 3-velocity is from $v_{0}=0.3 c$ to $v_{0}=0.98 c$, where $c$ is speed of light. The specific internal energy is varied from $\epsilon_{0} / c^{2}=0.1$ to $\epsilon_{0} / c^{2}=30$. The outflow of the model $\left(\Gamma_{0}, \epsilon_{0}\right)=(30,5)$ is similar to the models used by Zhang et al. (2003, 2004) and Umeda et al. (2005). This is the most attractive model for GRBs, since the outflow contains a large amount of thermal energy. Such an outflow could be formed in the quickly rotating progenitor. The outflow of the model $\left(\Gamma_{0}, \epsilon_{0} / c^{2}\right)=(1.05,0.1)$ is the most slowest and coldest one. The mass density of the injected outflow in model $\left(\Gamma_{0}, \epsilon_{0} / c^{2}\right)=(1.05,0.1)$ is $\sim 10^{4} \mathrm{~g} \mathrm{~cm}^{-3}$ and the highest one in all models. To the contrary, the mass density of the injected outflow in model $\left(\Gamma_{0}, \epsilon_{0} / c^{2}\right)=(5,30)$ is $\sim 1 \mathrm{~g} \mathrm{~cm}^{-3}$ and the lowest one in all models. As the mass density of the progenitor (before an outflow comes) around the injection point is $10^{6} \mathrm{~g} \mathrm{~cm}^{-3}$, the outflows in the all models are so-called "light jet" whose mass density is lower than that of the ambient gas. Thus we can expect strong interaction between the outflow and progenitor gas.

\section{Results and discussions}

Figure 1 shows the density (top) and Lorentz (bottom) contours of two models [left panel : $\left(\Gamma_{0}, \epsilon_{0} / c^{2}\right)=(5,30)$ and right panel : $\left.\left(\Gamma_{0}, \epsilon_{0} / c^{2}\right)=(1.05,0.1)\right]$, when the outflow breaks out from the progenitor surface. The outflow of the former model keeps good collimation in the progenitor, since the high Lorentz factor is localized along the cylindrical axis. To the contrary, the outflow of the latter model shows expanding feature. In both cases, the bow shock which drives progenitor 


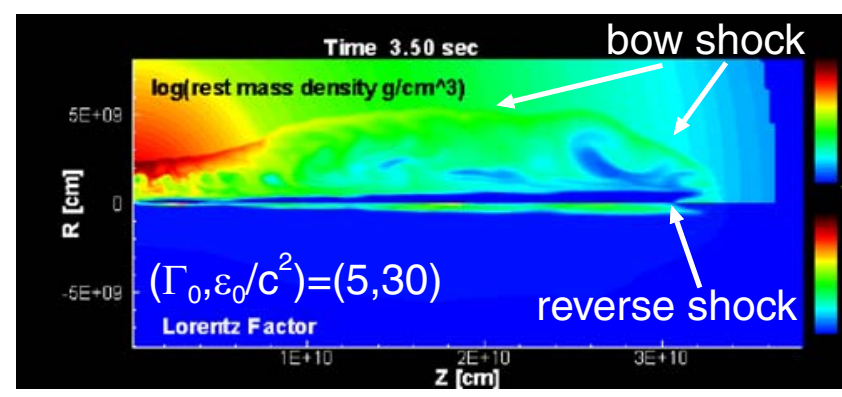

Fig. 1 The contours of rest mass density (top) and Lorentz factor (bottom) of two modes. Left panel shows collimated jet at $t=3.5$ $\mathrm{s}$ for case $\left[\left(\Gamma_{0}, \epsilon_{0} / c^{2}\right)=(5,30)\right]$. Right panel shows expanding out-

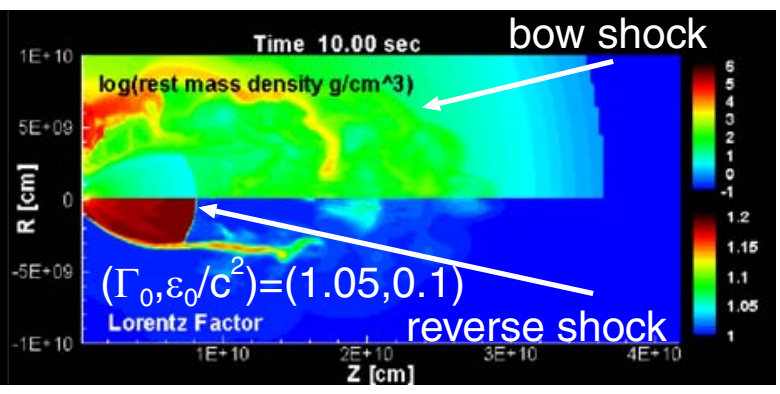

flow at $t=10 \mathrm{~s}$ for case $\left[\left(\Gamma_{0}, \epsilon_{0} / c^{2}\right)=(1.05,0.1)\right]$ Figures are taken from Mizuta et al. (2006) and reproduced by permission of the AAS.
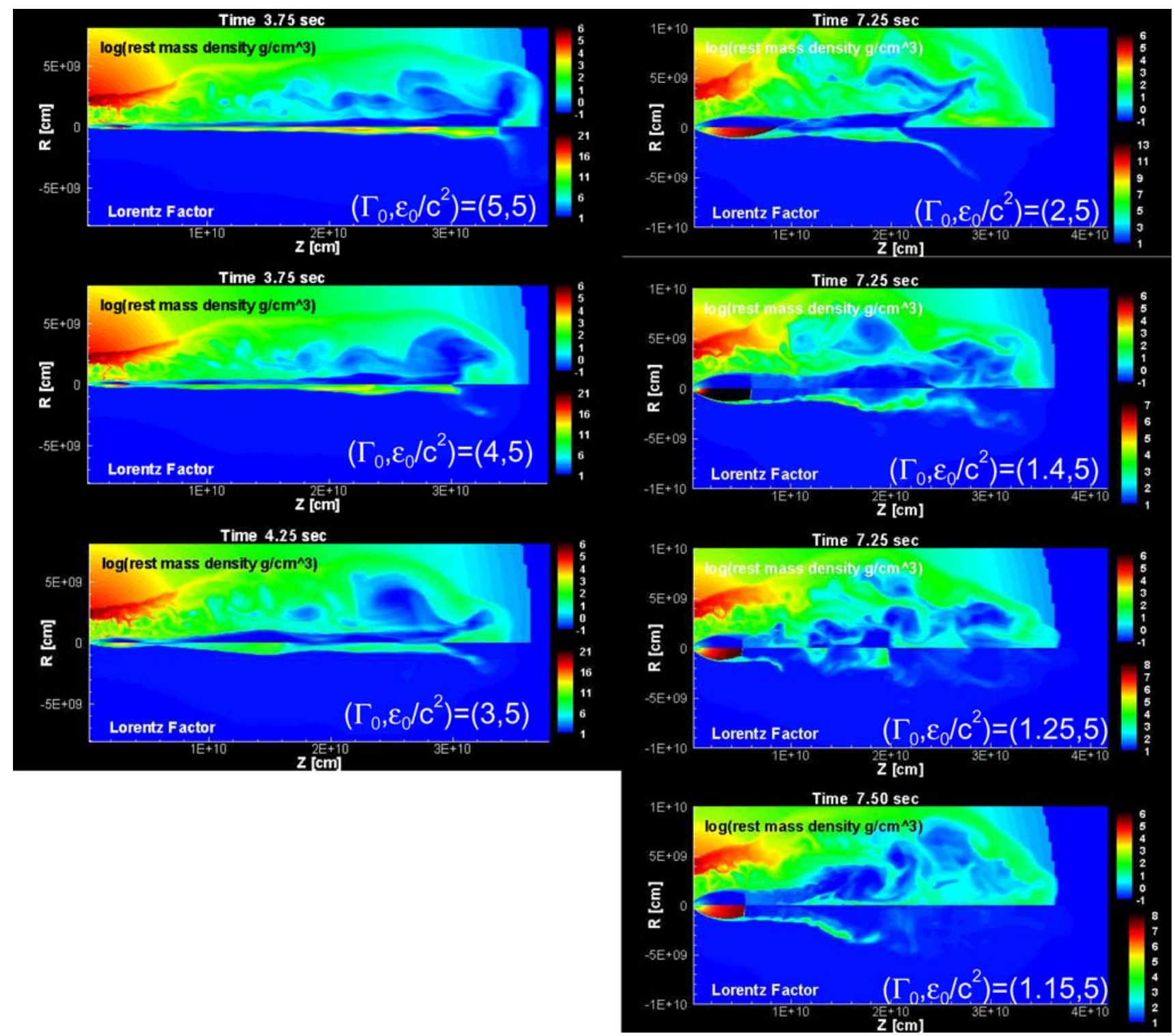

Fig. 2 The results of a series of calculations in which $\epsilon_{0} / c^{2}$ is fixed to be 5 . Models $\left(\Gamma_{0}, \epsilon\right)=(5,5),(4,5),(3,5),(2,5),(1.4,5),(1.25,5)$ and $(1.15,5)$ are shown The contours of the rest mass density and
Lorentz factor in each models are presented as same as in Fig. 1. Figures are taken from Mizuta et al. (2006) and reproduced by permission of the AAS. 
gas to high pressure and temperature can be seen. Since the bow shock is enough strong, the pressure driven by the bow shock can keep the outflow to be collimated structure in case of the collimated jet. The reverses shocks also appear in both models. In case of the collimated jet this shock is close to the bow shock, and located at the point where the bulk Lorentz factor decreases to unity. To the contrary, in case of expanding outflow, the distance between the bow shock and reverse shock increases as time goes on.

A back flow which is an anti-parallel flow to the main jet is observed in case of the collimated jet. This back flow begins from the shock heated gas through the reverses shock. Internal oblique shocks appear in the collimated jet which helps the jets to keep the collimated structure during the propagation in the progenitor (Norman, 1982; Falle, 1991; Leahy, 1991). There are two possibilities to appear such internal structures. The first is the dynamical nonlinear effect of Kelvin-Helmholtz instability which occurs at the boundary of the jet and the back flow. The second is the shear flow instability which occurs in the jet itself (Urpin, 2002). We need to do higher resolution calculations to identify the reason of the internal structures. No back flow is observed in case of the expanding outflow. In model $\left(\Gamma_{0}, \epsilon_{0} / c^{2}\right)=(5,30)$, the Lorentz factor increases up to 34 during the propagation in the progenitor, and to more than 100 after the break. The narrow opening angle for high Lorentz factor cases is good agreement with theoretical estimate of the opening angle $\sim 1 / \Gamma$. The appearance of such a high Lorentz factor component corresponds to the feature of the GRBs. This acceleration is caused by the energy conversion from the thermal energy to kinetic one. Since the outflow of model $\left(\Gamma_{0}, \epsilon_{0} / c^{2}\right)=(1.05,0.1)$ does not include so much thermal energy, no large acceleration is occurs. The flow is non-relativistic one.

Figure 2 shows the results of a series of the calculations, fixing $\epsilon_{0} / c^{2}=5$ and various $\Gamma_{0}$. The feature of the outflow changes from the collimated jet to the expanding outflow by decreasing the Lorentz factor of the injected outflow, i.e., $\Gamma_{0}$. The maximum Lorentz factor seen in each model also decreases from relativistic regime to non-relativistic regime by decreasing the $\Gamma_{0}$. A same continuous transition by changing the $\Gamma_{0}$ is observed in the series of the calculations in which $\epsilon_{0} / c^{2}$ is fixed to be 1 or 0.1 (Mizuta et al., 2006).

We have observed different types of the outflow propagation in the progenitor. The outflows which can keep collimated structure and becomes high Lorentz factor would be observed as GRBs, since the properties correspond to those of GRBs. Even if the outflows keeps collimated structure, the Lorentz factor increases up to a few in some models. Such outflows could be observed as XRFs. The outflows which do not keep good collimation but are mildly relativistic flows also would be the candidate of XRFs. The outflows which have large opening angle and expanding features would be observed as aspherical $\mathrm{SNe}$ (no accompanied GRBs).
Recently several types of laboratory experiments to produce jet like flows have been proposed and done by using laser produced plasmas (Farley et al., 1999; Shigemori et al., 2000; Mizuta et al., 2002; Foster et al.,2005) and Z-pinch plasmas (Lebedev et al., 2002). Those are usually dense outflows and suitable to study the dynamics of protostar jets. Wheres the all outflows presented in this paper are light jet which shows a variety of properties of morphology and dynamics. We hope that we can produce such light jets in the laboratory to study the different type of the morphology and dynamics shown in this paper in the near future.

\section{Conclusion}

We investigate the outflow propagation in the collapsar in the context of gamma-ray bursts (GRBs) with 2D relativistic hydrodynamic simulations. We observed a variety of the outflow properties by changing the specific internal energy and bulk Lorentz factor of the injected outflow from nonrelativistic regime to relativistic one. The feature of the outflow changes from the collimated jets to expanding outflows by decreasing the $\Gamma_{0}$. The observed different features of the dynamics possibly explain the different features of the similar phenomena such as, GRBs and XRFs. The production of the light jet in the laboratory is expected to study the features observed in this study.

Acknowledgment This work was carried out on NEC SX5, Cybermedia Center and Institute of Laser Engineering, Osaka University, and Fujitsu VPP5000 of National Observatory of Japan. This work was supported in part by the Grants-in-Aid of the Ministry of Education, Science, Culture, and Sport (14079205, A.M., S.M.) and (14102004, 14079202 , and 16740134 , S.N.), This work was supported by the Grantin-Aid for the 21st Century COE "Center for Diversity and Universality in Physics" from the Ministry of Education, Culture, Sports, Science and Technology (MEXT) of Japan.

\section{References}

Aloy, M.A., Müller, E., Ibáñez, J.M., Martí, J.M., MacFadyen, A.: ApJ 531, L119 (2000)

Falle, S.A.E.G.: MNRAS 250, 581 (1991)

Farley, D.R. et al.: PRL 83, 1982 (1999)

Foster, J.M., et al.: ApJ 634, L77 (2005)

Galama, T.J. et al.: Nature 395, 670 (1998)

Hashimoto, M.: Progress of Theoretical Physics 94, 663 (1995)

Heise, J., in't Zand, J., Kippen, R.M., Woods, P.M.: Gamma-ray Bursts in the Afterglow Era, 16 (2001)

Hjorth, J. et al.: Nature 423, 847 (2003)

Iwamoto, K. et al.: Nature 395, 672 (1998)

Lamb, D.Q., Donaghy, T.Q., Granziani, C.: Il Nuovo Cimento (astro$\mathrm{ph} / 0505156)(2005)$

Leahy, J.P.: In: Hughes, P.A. (ed.), Beams and jets in astrophysics. Cambridge Astrophysics Series, No. 19. Cambridge, UK: Cambridge University Press, 100 (1991) 
Lebedev, S.V. et al.: ApJ 564, 113 (2002)

MacFadyen, A.I., Woosley, S.E.: ApJ 524, 262 (1999)

Mizuta, A., Yamada, S., Takabe, H.: ApJ 567, 635 (2002)

Mizuta, A., Yamada, S., Takabe, H.: ApJ 606, 804 (2004)

Mizuta, A. et al.: ApJ 651, 960 (2006)

Nakamura, T.: ApJ 534, L159 (2000)

Norman, M.L., Winkler, K.-H.A., Smarr, L., Smith, M.D.: A\&A 113, 285 534, L159 (1982)
Piran, T.: Phys. Rep. 333, 529 (2000)

Price, P.A. et al.: Nature 423, 844 (2003)

Shigemori, K. et al.: PRE 62, 8838 (2000)

Stanek, K.Z. et al.: ApJ 591, L17 (2003)

Umeda, H., Tominaga, N., Maeda, K., Nomoto, K.: ApJL 633, L17 (2005)

Urpin, V.: A\&A 385, 14 (2002)

Woosley, S.E.: ApJ 405, 273 (1993) 